\title{
ON THE INERTIA GROUPS OF $h$-COBORDANT MANIFOLDS
}

\author{
MICHAEL FRAME ${ }^{1}$
}

\begin{abstract}
It is shown that if $M$ and $N$ are $h$-cobordant manifolds of dimension at least eight, then their special inertia groups are equal. (G. Brumfiel has shown that special inertia groups are not homotopy type invariants.) Examples are constructed for which the inertia group is an $h$-cobordism invariant.
\end{abstract}

Introduction. Recall that an exotic $n$-sphere is a smooth manifold homeomorphic to $S^{n}$. It was shown by $\mathbf{J}$. Milnor [15] that such manifolds need not be diffeomorphic to $S^{n}$, hence the term "exotic spheres", and the group (under connected sum \#) of oriented exotic $n$-spheres is denoted $\Theta_{n}$. Letting $\Gamma^{n}=\pi_{0} \operatorname{Diff}\left(S^{n-1}\right)$, the map $\Gamma^{n} \rightarrow \Theta_{n}$ given by $[f] \mapsto D^{n} \cup{ }_{f} D^{n}=\Sigma(f)$ is an isomorphism for $n \geqslant 5$ [15]. The inertia group $I(M)$ of a smooth $n$-manifold $M$ is defined as $\left\{\Sigma \in \Theta_{n}: \Sigma \# M \cong M\right\}$, and it is a consequence of the Disc Theorem of $J$. Cerf and R. Palais that $\Sigma(F) \in I(M)$ if and only if there is a diffeomorphism $F: M^{n} \backslash$ int $D^{n} \rightarrow M^{n} \backslash$ int $D^{n}$ satisfying $F \mid \partial D^{n}=f$. Thus $I(M)$ can be viewed as a measure of the stability of the differentiable structure of $M$ under perturbations on an $n$-disc.

Definition. Writing $M \# \Sigma(g)$ as $\left(M^{n} \backslash\right.$ int $\left.D^{n}\right) \cup_{g} D^{n}$, let $\iota: M \rightarrow M \# \Sigma(g)$ denote the PL homeomorphism defined by $\iota \mid M \backslash$ int $D^{n}=$ identity and $\iota \mid D^{n}=c(g)$ = cone map on $g$. (The letter $\iota$ will be used for different $M$; which manifold is involved will be clear from the context.)

In [4] a special inertia group $I_{h}(M)$ is defined as $\left\{\Sigma \in \Theta_{n}\right.$; there is a diffeomorphism $f: M \rightarrow M \# \Sigma$ homotopic to $\iota$.

R. DeSapio [6], K. Kawakubo [13] and R. Schultz [17] independently showed $I(M)$ is not a homotopy type invariant of $M$. They showed $I\left(S^{p} \times S^{q}\right)=0$ and yet for some exotic spheres $\Sigma^{p}$ and for some $q, I\left(\Sigma^{p} \times S^{q}\right) \neq 0$. Since $\Sigma^{p}$ is PL homeomorphic to $S^{p}$, this also showed $I(M)$ is not a PL invariant of $M$.

In Proposition II.1 of [4] G. Brumfiel computes $I_{h}(M)$ for simply-connected $M$ via D. Sullivan's theory of homotopy smoothings. In Remark II.12 Brumfiel shows there are manifolds $P_{1}$ and $P_{2}$, homotopy equivalent to $\mathrm{CP}(3)$, with $I_{h}\left(P_{1} \times S^{1}\right)=0$ and $I_{h}\left(P_{2} \times S^{1}\right)=\mathbf{Z}_{7}$. Thus $I_{h}(M)$ is not a homotopy type invariant of $M$.

The goal of this paper is to show that $I_{h}(M)$ is an $h$-cobordism invariant for manifolds of dimension at least eight. Examples are constructed for which $I(M)$ is

Received by the editors September 9, 1982 and, in revised form, April 11, 1983.

1980 Mathematics Subject Classification. Primary 57R55; Secondary 57R80.

$K e y$ words and phrases. Exotic sphere, special inertia group, $h$-cobordism.

'The author was partially supported by Grant Number A7579 of the National Research Council of Canada. 
an $h$-cobordism invariant of $M$. It is a pleasure to acknowledge helpful conversations with Ian Hambleton, Terry Lawson, Youn Lee and Brian Mortimer. In addition, the referee's useful comments are appreciated.

\section{Some special cases.}

Proposition 1. Let $\left(W^{n+1} ; M, N\right)$ be an h-cobordism with $n \geqslant 5$ and let $\sigma=$ $\tau(W, M) \in \mathrm{Wh}\left(\pi_{1} M\right)$. For $\Sigma \in I(M)$, if there is a diffeomorphism $f: M \rightarrow M \# \Sigma$ with $f_{*} \sigma=\iota_{*} \sigma$, then $\Sigma \in I(N)$.

Proof. Let $V$ denote the connected sum along the cobordism of $W$ with $\Sigma \times I$, so $(V ; M \# \Sigma, N \# \Sigma)$ is an $h$-cobordism. Observe that $\tau(V, M \# \Sigma)=\iota_{*} \sigma \in$ $\mathrm{Wh}\left(\pi_{1} M \# \Sigma\right)$. Since $f_{*} \sigma=\iota_{*} \sigma$, it follows from the uniqueness theorem for $h$-cobordisms (11.3 of [16]) that $f$ extends to a diffeomorphism $W \rightarrow V$, so $N \cong N \# \Sigma$.

COROLlARY 1. If $\pi_{1} M$ admits only inner automorphisms, then $I(M)=I(N)$ for any h-cobordism $\left(W^{n+1} ; M, N\right)$ with $n \geqslant 5$.

Proof. For any diffeomorphism $f: M \rightarrow M \# \Sigma,\left(f^{-1} \iota\right)_{\#}$ induces an inner automorphism on $\pi_{1} M$ and so, by 6.1 of [16], $f_{*}=\iota_{*}$ on $\mathrm{Wh}\left(\pi_{1} M\right)$.

EXAMPLE 1. If $\pi_{1} M$ is complete, then $I(M)=I(N)$. Z. Janko's sporadic finite simple group $J_{1}$ is complete [12] and by a theorem of $\mathrm{H}$. Bass $\left(6.2\right.$ of [15]) $\mathrm{Wh}\left(J_{1}\right)$ has rank five.

COROllary 2. Let $\left(W^{n+1} ; M, N\right)$ be an h-cobordism with $n \geqslant 5$. Then $I_{h}(M) \subset$ $I(N)$.

Proof. If $\Sigma \in I_{h}(M)$, then $\iota \simeq f: M \rightarrow M \# \Sigma$ and so $f_{*}=\iota_{*}$ on Whitehead groups.

More work is required to show $\Sigma \in I_{h}(N)$ (see Corollary 2 of $\S 5$ ), but an attempt can be made to modify this argument and compare $I_{h}(M)$ with $I_{h}(N)$.

Corollary 3. Let $\left(W^{n+1} ; M, N\right)$ be an h-cobordism with $n \geqslant 5$ and odd, and suppose conjugation ( see p. 373 of [16]) is trivial in $\mathrm{Wh}\left(\pi_{1} M\right)$. Then $I_{h}(M)=I_{h}(N)$.

Proof. Take $\Sigma \in I_{h}(M)$ and $f: M \rightarrow M \# \Sigma$ homotopic to $\iota$. As above, let $V$ denote the connected sum along the cobordism of $W$ with $\Sigma \times I$, and let $(Y ; N, N \# \Sigma$ ) denote the $h$-cobordism $\bar{W} \cup, V$, where $(\bar{W} ; N, M)$ is the reflection of $(W ; M, N)$ (see 11.5 of [16]). Measuring torsions in the middle $\mathrm{Wh}\left(\pi_{1} M\right)$, it follows that

$$
\begin{aligned}
\tau(Y, N) & =\tau(\bar{W}, N)+f_{*}^{-1} \tau(V, M \# \Sigma)=\tau(\bar{W}, N)+f_{*}^{-1} \iota_{*} \tau(W, M) \\
& =(-1)^{n} \bar{\tau}(W, M)+\tau(W, M)=-\tau(W, M)+\tau(W, M)=0 .
\end{aligned}
$$

The s-cobordism theorem implies there is a trivialization $g: Y \rightarrow N \times I$. From $g$ and the homotopy between $f$ and $\iota$, a homotopy between $\left(g \mid \partial_{+} Y\right)^{-1}: N \rightarrow N \# \Sigma$ and $\iota$ can be constructed, so $\Sigma \in I_{h}(N)$.

EXAmple 2. Conjugation is trivial in $\mathrm{Wh}\left(\mathbf{Z}_{q}\right)$ (see Theorem 1 of [1] or Proposition 4.2 of [3]). If $\left(W ; L_{q}, M\right)$ is an $h$-cobordism, $L_{q}$ a $(2 n+1)$-dimensional lens space, and $\tau\left(W, L_{q}\right) \neq 0$, then $L_{q} \not M$ (Corollary 12.13 of [16]) and so lens spaces $L_{q}$ provide examples of $h$-cobordisms which $L_{q} \not M$ and $I_{h}\left(L_{q}\right)=I_{h}(M)$. (Examples 
with nonzero torsion abound: recall $\mathrm{Wh}\left(\mathbf{Z}_{q}\right) \neq 0$ if $\left.q \neq 1,2,3,4,6\right)$. In many instances it is easy to see that $I_{h}\left(L_{q}\right)$-in fact, $I\left(L_{q}\right)$-cannot be all of $\Theta_{n}$. A diffeomorphism $\Sigma \# L_{q} \rightarrow L_{q}$ lifts to a diffeomorphism of universal covers $q \sum \# S^{2 n+1}$ $\rightarrow S^{2 n+1}$. Since $I\left(S^{2 n+1}\right)=0$, it follows that the order of $\Sigma$ must divide $q$.

EXAmple 3 . If $p \equiv 3(\bmod 4), k \geqslant 3$, and $M$ is $h$-cobordant to $L_{p}^{3} \times S^{2 k}$, then Theorem 6.1 of [10] shows $L_{p}^{3} \times S^{2 k} \not M$ and yet by Corollary 3 above, $I_{h}(M)=$ $I_{h}\left(L_{p}^{3} \times S^{2 k}\right)$. Since $I\left(S^{3} \times S^{2 k}\right)=0$, an argument analogous to that given in Example 2 shows the order of $\Sigma$ must divide $p$ if $\Sigma \in I\left(L_{p}^{3} \times S^{2 k}\right)$.

3. Inertia groups of connected sums. For all $n$-manifolds $M$ and $N$, the Disc Theorem shows that a diffeomorphism $f: N \rightarrow N \# \Sigma$ extends to a diffeomorphism $f \# 1: N \# M \rightarrow(N \# \Sigma) \# M$. In fact, one has $I(N)+I(M) \subset I(N \# M)$. To show that inclusion is sometimes strict, D. Wilkens [18] constructed 15-manifolds $M$ and $N$ with $I(M)=I(N)=0$ and $I(M \# N)=\mathbf{Z}_{127}$.

As a model for the proof of the theorem $(\S 5)$, the behavior of $I_{h}$ with respect to connected sum will be studied.

Proposition 2. For all $n$-manifolds $N$ and $M, n \geqslant 5, I_{h}(M) \subset I_{h}(N \# M)$.

Proof. Take $\Sigma \in I_{h}(M)$ and let $F: M \times I \rightarrow(M \# \Sigma) \times I$ be the track of a homotopy with $F_{1}$ a diffeomorphism and $F_{0}=\imath$. Writing $M \# \Sigma$ as $\left(M \backslash\right.$ int $\left.D^{n}\right) \cup_{f} D^{n}$ and $M$ as $\left(M \backslash\right.$ int $\left.D^{n}\right) \cup_{\text {id }} D^{n}$, and selecting $x_{0} \in M \backslash D^{n}$, the diffeomorphism $F_{1}$ can be isotoped to fix $x_{0}$. After modification by a homotopy, $F$ can be taken to be smooth on a neighhorhood of $x_{0} \times I$. Let $p$ denote the projection $(M \# \Sigma) \times I \rightarrow$ $(M \# \Sigma) \times\{1\}$. By transversality, $F \mid x_{0} \times I$ and $p F \mid x_{0} \times I$ can be taken to be smooth imbeddings. In $(M \# \Sigma) \times I$ the circle $\left(x_{0} \times I\right) \cup F\left(x_{0} \times I\right) \cup p F\left(x_{0} \times I\right)$ bounds a $D^{2}$ (imbedded since $n \geqslant 5$ ), which can be used to pseudo-isotope $F \mid x_{0} \times I$, relative to $x_{0} \times 0$, to the inclusion. Then by Theorem 2.1 of [11] and isotopy extension, there is a diffeomorphism $H:(M \# \Sigma) \times I \rightarrow(M \# \Sigma) \times I$ satisfying $H F$ $x_{0} \times I=$ inclusion and $H \mid(M \# \Sigma) \times 0=$ identity.

Let $D(t)$ denote the derivative of $H F$ at the point $\left(x_{0}, t\right)$. Then $D(0)$ and $D(1)$ are invertible, so by the continuity of the determinant $D(t)$ is invertible for $0 \leqslant t<\varepsilon$ and $1-\varepsilon<t \leqslant 1$. Modifying $H F$ on an arbitrarily small neighborhood of $x_{0} \times[\varepsilon, 1$ $-\varepsilon], D(t)$ is invertible for all $t \in[0,1]$. The inverse function theorem implies that for some small $n$-disc $A$ around $x_{0}, H F \mid A \times I$ is an imbedding, hence provides a framing for $A \times I \subset(M \# \Sigma) \times I$ differing from the standard framing $T=D^{n} \times I$ $\rightarrow \nu\left(x_{0}\right) \times I \subset(M \# \Sigma) \times I$ by a bundle diffeomorphism determined by $\lambda \in$ $[(I, \partial I),(\mathrm{SO}(n),[\mathrm{id}])]$. Let $S: D^{n} \times I \rightarrow \nu\left(x_{0}\right) \times I \subset M \times I$ denote the standard framing. Then $T^{-1} H F S(x, t)=(\lambda(t) \cdot x, t)$, and the fact that $\lambda(0)=\lambda(1)=[$ id] can be used to produce a self-diffeomorphsim $E_{1}$ of $D^{n} \times I$ satisfying $E_{1} \mid\left(\partial D^{n} \times I\right) \cup$ $\left(1 / 2 D^{n} \times 0\right)=$ inclusion and $E_{1}\left|1 / 2 D^{n} \times I=T^{-1} H F S\right| 1 / 2 D^{n} \times I$. (Specifically, let $\alpha=[0,1] \rightarrow[0,1]$ be a smooth map satisfying $\alpha(s)=1$ if $0 \leqslant s \leqslant \frac{1}{2}$ and $\alpha(1)=0$. Then $E_{1}$ is given by $E_{1}(x, t)=(\lambda(\alpha(\|x\|) t) \cdot x, t)$.) Thus $T E_{1} T^{-1}$ extends by the identity to a diffeomorphism $E_{2}$ of $(M \# \Sigma) \times I$. The composition $E_{2}^{-1} H F$ is the ideniity along $A \times I$, hence extends by the identity to a homotopy between a diffeomorphism $M \# N \rightarrow(M \# \Sigma) \# N$ and $\iota$. This completes the proof. 
4. Stably diffeomorphic manifolds. One method of studying $h$-cobordant manifolds is the following well-known result (see the proof of the Proposition of [8]).

LEMma 1. If $M^{n}$ is h-cobordant to $N^{n}$ and $n \geqslant 5$, then for sufficiently large $m$ there is a diffeomorphism $M \# m\left(S^{2} \times S^{n-2}\right) \cong N \# m\left(S^{2} \times S^{n-2}\right)$.

Proof. Any $h$-cobordism between $M$ and $N$ can be constructed by trivially attaching $m$ 2-handles to $M \times I$ and then attaching $m$ 3-handles to obtain the correct torsion (see Theorem 11.1 of [16]). Since the 2-handles are attached trivially, a level surface between the 2-handles and the 3-handles is $M \# m\left(S^{2} \times S^{n-2}\right)$. In the dual handlebody decomposition of the $h$-cobordism, one starts with $N \times I$, attaches $m(n-2)$-handles trivially, and then attaches $m(n-1)$-handles to obtain the correct torsion. A level surface between the $(n-2)$-handles and the $(n-1)$-handles is $N \# m\left(S^{2} \times S^{n-2}\right)$. This completes the proof.

Let $M$ and $N$ be $h$-cobordant $n$-manifolds, $n \geqslant 5$, and let $g: M \# m\left(S^{2} \times S^{n-2}\right) \rightarrow$ $N \# m\left(S^{2} \times S^{n-2}\right)$ be the diffeomorphism given by Lemma 1. If $\Sigma \in I(N)$, there is a diffeomorphism $h: N \rightarrow N \# \Sigma$ and the composition $f$ given by

$$
\begin{aligned}
M \# m\left(S^{2} \times S^{n-2}\right) & \stackrel{g}{\rightarrow} N \# m\left(S^{2} \times S^{n-2}\right) \\
& \stackrel{h \# 1}{\rightarrow} \Sigma \# N \# m\left(S^{2} \times S^{n-2}\right) \stackrel{1 \# g^{-1}}{\rightarrow} \Sigma \# M \# m\left(S^{2} \times S^{n-2}\right)
\end{aligned}
$$

shows $\Sigma \in I\left(M \# m\left(S^{2} \times S^{n-2}\right)\right)$. The argument to show $\Sigma \in I(M)$ proceeds by modifying $f$ so successive summands of $S^{2} \times S^{n-2}$ can be removed by surgery on $S^{2}$.

LEMMA 2. If there is a diffeomorphism $f: M_{0}=M^{n} \#\left(S^{2} \times S^{n-2}\right) \rightarrow N^{n} \#\left(S^{2} \times\right.$ $\left.S^{n-2}\right)=N_{0}$ satisfying $f \mid S^{2} \simeq$ inclusion for $S^{2}=S^{2} \times\{*\} \subset S^{2} \times S^{n-2}$, and if $n \geqslant$ 7, then there is a diffeomorphism $M \rightarrow N$.

PROOF. The result will follow by a surgery on $S^{2} \times D^{n-2} \subset S^{2} \times S^{n-2}$ if it can be shown that $f$ fixed this $S^{2} \times D^{n-2}$. By transversality $f\left(S^{2}\right)$ can be taken to miss $S^{2}$ and the homotopy $S^{2} \times I \rightarrow N_{0}$ between $f \mid S^{2}$ and the inclusion can be taken to be an imbedding (since $n \geqslant 7$ ), hence a pseudo-isotopy. Theorem 2.1 of [11] implies $f \mid S^{2}$ is isotopic to the inclusion, so by isotopy extension there is a diffeomorphism $f^{\prime}: M_{0} \rightarrow N_{0}$ with $f^{\prime} \mid S^{2} \simeq$ inclusion.

Let $\nu_{M}$ denote the normal bundle $\nu\left(S^{2} \rightarrow M_{0}\right)$ and let $\nu_{N}$ denote $\nu\left(S^{2} \rightarrow N_{0}\right)$. Then by the tubular neighborhood theorem $f^{\prime}$ can be isotoped to $f^{\prime \prime}$ satisfying $f^{\prime \prime}\left(\nu_{M}\right)=\nu_{N}$ and $f^{\prime \prime} \mid \nu_{M}$ is a vector bundle isomorphism. Since $\pi_{2} \mathrm{SO}(n-2)=0, f^{\prime \prime}$ can be isotoped to give the standard identification on a neighborhood $S^{2} \times D^{n-2}$ of $S^{2}$. Surgery on this framed $S^{2}$ gives the result.

COROLlaRY 1. If $M^{n}$ is 2-connected, $n \geqslant 7$, and if there is a diffeomorphism $f$ : $M \# m\left(S^{2} \times S^{n-2}\right) \rightarrow N \# m\left(S^{2} \times S^{n-2}\right)$, then there is a diffeomorphism $M \cong N$.

Proof. The result follows from $m$ applications of Lemma 2, provided there is a diffeomorphism $h$ of $N \# m\left(S^{2} \times S^{n-2}\right)$ with $h f \mid+m S^{2} \simeq$ inclusion. Since $\pi_{2}\left(N \# m\left(S^{2} \times S^{n-2}\right)\right) \cong \oplus m\left\langle S^{2}\right\rangle \cong \mathbf{Z}^{m}$, it suffices to show any element of 
$\operatorname{Aut}\left(\mathbf{Z}^{m}\right)=\mathrm{GL}(m, \mathbf{Z})$ can be induced by a diffeomorphism of $\# m\left(S^{2} \times S^{n-2}\right)$. Any element of $\operatorname{GL}(m, \mathbf{Z})$ can be written as a product of permutation matrices and "elementary" matrices (the identity matrix with \pm 1 in one off-diagonal location). It suffices to give a diffeomorphism $g$ of $\left(S_{1}^{2} \times S^{n-2}\right) \#\left(S_{2}^{2} \times S^{n-2}\right)$, inducing the automorphism $\left(\begin{array}{ll}1 & 1 \\ 0 & 1\end{array}\right)$ on $\pi_{2}$. Split $\left(S_{1}^{2} \times S^{n-2}\right) \#\left(S_{2}^{2} \times S^{n-2}\right)$ as $A \cup_{1} A$, where $A=S_{1}^{2} \times D^{n-2} \Perp S_{2}^{2} \times D^{n-2}$, and observe that $A \cup_{1} A$ admits an involution $t$ interchanging the copies of $A$ and fixing $\partial A$ pointwise. Construct a diffeomorphism $h$ : $A \rightarrow A$ as follows. Send disjoint neighborhoods of $S_{1}^{2}$ and $S_{2}^{2}$ to disjoint neighborhoods of $S_{1}^{2}+S_{2}^{2}$ and $S_{2}^{2}$, respectively. Connect each pair of neighborhoods by a neighborhood of an arc joining $S_{1}^{2}$ and $S_{2}^{2}$. Then the remainder of the domain is $\partial A \times I$, as is the remainder of the range (by the $h$-cobordism theorem). Extend the given map to produce $h$, and extend $h$ to the second copy of $A$ by $t h t$. This yields $g$.

COROLLARY 2. If $M^{n}$ is 2-connected and $n \geqslant 7$, then for all $m$,

$$
I(M)=I\left(M \# m\left(S^{2} \times S^{n-2}\right)\right) \text {. }
$$

Proof. Suppose g: $(\Sigma \# M) \# m\left(S^{2} \times S^{n-2}\right) \rightarrow M \# m\left(S^{2} \times S^{n-2}\right)$. Then Corollary 1 implies there is a diffeomorphism $\Sigma \# M \rightarrow M$, so $I\left(M \# m\left(S^{2} \times S^{n-2}\right)\right)$ $\subset I(M)$. The reverse inclusion follows from the remark at the beginning of $\S 3$.

The connectivity assumptions of Corollaries 1 and 2 are necessary in order to homotope $f\left(+m S^{2}\right)$ into $\# m\left(S^{2} \times S^{n-2}\right)$ because otherwise $f\left(+m S^{2}\right) \cap M$ may have annular components which are nontrivial in $\pi_{1}\left(M \backslash\right.$ int $\left.D^{n}, \partial\right)$ or disc components which are nontrivial in $\pi_{2}\left(M \backslash\right.$ int $\left.D^{n}, \partial\right)$. In some specific instances, however, the connectivity hypotheses can be removed.

EXAMPLE 4. Let $\left(W, L_{p}^{3} \times S^{2 k}, N\right)$ be an $h$-cobordism with nontrivial torsion and let $p \equiv 3(\bmod 4)$ and $k \geqslant 3$. By Theorem 6.1 of [10], $N \not L_{p}^{3} \times S^{2 k}$. In Example 3 it was shown that $I_{h}(N)=I_{h}\left(L_{p}^{3} \times S^{2 k}\right)$. Lemma 2 can be used to show $I(N)=$ $I\left(L_{p}^{3} \times S^{2 k}\right)$. Given a diffeomorphism $h: L_{p}^{3} \times S^{2 k} \rightarrow \Sigma \#\left(L_{p}^{3} \times S^{2 k}\right)$, construct

$$
f=(1 \# g)(h \# 1) g^{-1}: N \# m\left(S^{2} \times S^{2 k+1}\right) \rightarrow \Sigma \# N \# m\left(S^{2} \times S^{2 k+1}\right)
$$

as above. Since $\pi_{2}\left(\left(L_{p}^{3} \times S^{2 k}\right) \# m\left(S^{2} \times S^{2 k+1}\right)\right)=\oplus m \mathbf{Z}\left[\mathbf{Z}_{p}\right]$, generated by the inclusions of the $m$ factors of $S^{2}, h \# 1$ induces the identity on $\pi_{2}$. Consequently, $\iota^{-1} f$ also induces the identity on $\pi_{2}$, and $m$ applications of Lemma 2 imply $I\left(L_{p}^{3} \times S^{2 k}\right)$ $\subset I(N)$. The reverse inclusion is given by a similar argument.

EXAMPLE 5. In Example 2 it was shown that $I_{h}(L)=I_{h}(N)$ if $N^{2 n+1}$ is $h$-cobordant to a lens space $L$. Since $\pi_{2}\left(L \# m\left(S^{2} \times S^{2 n-1}\right)\right) \cong \bigoplus m \mathbf{Z}\left[\mathbf{Z}_{p}\right]$, the argument of Example 4 can be rephrased to show $I(N)=I(L)$.

6. Stable diffeomorphisms homotopic to $\iota$. The comparison of special inertia groups of $h$-cobordant manifolds is made by the following result.

THEOREM. If a diffeomorphism $f: M \#\left(S^{2} \times S^{n-2}\right) \rightarrow \Sigma \# M \#\left(S^{2} \times S^{n-2}\right)$ satisfies $f \simeq \iota$, and if $n \geqslant 8$, there is a diffeomorphism $g: M \rightarrow \Sigma \# M$ satisfying $g \simeq \iota$.

Proof. Let $S_{1}^{2}=S^{2} \times\{*\} \subset S^{2} \times S^{n-2}$ in the domain of $f$ and let $S_{2}^{2}=S^{2} \times\{*\}$ in the range of $f$. Isotope $f$ so that $f\left(S_{1}^{2}\right)$ misses $S_{2}^{2}$. Since $\iota \mid S_{1}^{2}=$ inclusion, the proof 
proceeds by applying Lemma 2 to produce a diffeomorphism $f^{\prime}: M \rightarrow M \# \Sigma$ and then extending the argument of Proposition 2 to show $f^{\prime} \cong \iota$. Let $F: A \times I \rightarrow B \times I$ be the track of a homotopy with $F_{1}=f$ and $F_{0}=\imath$, and take $F$ to be smooth near $S_{1}^{2} \times I$. By transversality $F$ can be taken to be an imbedding on $S_{1}^{2} \times I$. Then Theorem 2.1 of [11] and isotopy extension imply there is an isotopy $F^{\prime}: A \times[1,2] \rightarrow$ $B \times[1,2]$ with $F_{1}^{\prime}=f, F_{2}^{\prime}=f^{\prime}, f^{\prime}\left|S_{1}^{2}=\iota\right| S_{1}^{2}$, and $F^{\prime}\left|S_{1}^{2} \times\{t\}=F\right| S_{1}^{2} \times\{2-t\}$. Let $G: A \times I \rightarrow B \times I$ be the homotopy given by $G(a, t)=F(a, 2 t)$ for $0 \leqslant t \leqslant \frac{1}{2}$ and $G(a, t)=F^{\prime}(a, 2 t)$ for $\frac{1}{2} \leqslant t \leqslant 1$. Now $G \mid S_{1}^{2} \times I$ is homotopic, rel $S_{1}^{2} \times\{0,1\}$, to the inclusion, so by transversality (since $n \geqslant 8) G \mid S_{1}^{2} \times I$ is pseudo-isotopic to the inclusion. Theorem 2.1 of [11] and isotopy extension give rise to a level-preserving diffeomorphism $H: B \times I \rightarrow B \times I$ satisfying $H F \mid S_{1}^{2} \times I=$ inclusion and $H \mid B \times 0$ $=$ identity.

It remains to modify $H F$ to be the inclusion on a neighborhood of $S_{1}^{2} \times I$. Observe $\iota$ is the inclusion on a neighborhood of $S_{1}^{2} \times 0$ and since $\pi_{2} \operatorname{SO}(n-2)=0$, $(H F)_{1}=g$ can be taken to be the inclusion on a neighborhood of $S_{1}^{2} \times 1$. Let $D^{n-2} \times S_{i}^{2} \times I$ denote the standard framing of $\nu\left(S_{i}^{2} \times I \rightarrow A \times I\right)$. Recall $H F$ is the track of a homotopy-that is, $H F$ preserves the $I$-coordinate. Modify $H F$ on $D^{n-2} \times S_{1}^{2} \times I$, rel $\partial D^{n-2} \times S_{1}^{2} \times I$, in two stages. First, send $(x, y, t)$ to $(0, y, t)$ if $\|x\| \leqslant \frac{1}{2}$ and to $(x(2\|x\|-1), y, t)$ if $\frac{1}{2} \leqslant\|x\| \leqslant 1$. A map $D^{n-2} \times S_{1}^{2} \times I \rightarrow B \times I$ which agrees with $H F$ on $\partial D^{n-2} \times S_{1}^{2} \times I$ and which is the inclusion on $1 / 2 D^{n-2}$ $\times S_{1}^{2} \times I$ similarly can be shrunk to send $1 / 2 D^{n-2} \times S_{1}^{2} \times I$ to $0 \times S_{1}^{2} \times I$. The second modificiation of $H F$ is the reverse of this homotopy. Thus modified, $H F$ provides a homotopy between $\iota$ and a diffeomorphism $g$, and $H F$ restricts to the inclusion on $D^{n-2} \times S_{1}^{2} \times I$. Using this framing, surgery on $S_{1}^{2} \times I$ yields the result.

Corollary 1. If $n \geqslant 8$ then $I_{h}\left(M^{n}\right)=I_{h}\left(M^{n} \# m\left(S^{2} \times S^{n-2}\right)\right.$ ) for all $m$.

Proof. Proposition 2 implies $I_{h}(M) \subset I_{h}\left(M \# m\left(S^{2} \times S^{n-2}\right)\right)$. The reverse inclusion follows from $m$ applications of the theorem.

Corollary 2. If $M^{n}$ is $h$-cobordant to $N^{n}$ and $n \geqslant 8$, then $I_{h}(M)=I_{h}(N)$.

Proof. Lemma 1 implies that for sufficiently large $m, I_{h}\left(M \# m\left(S^{2} \times S^{n-2}\right)\right)=$ $I_{h}\left(N \# m\left(S^{2} \times S^{n-2}\right)\right)$. Corollary 1 implies $I_{h}(M)=I_{h}(N)$.

In a sequel this argument will be extended to study inertial $h$-cobordisms and diffeomorphism groups of $h$-cobordant manifolds.

This corollary shows that the pathology detected by Brumfiel in proving $I_{h}(M)$ is not a homotopy type invariant does not persist if "homotopy equivalent" is replaced by " $h$-cobordant".

ExAmple 6. In [7] F. Farrell and W.-C. Hsiang construct, for each $n \geqslant 6$, $h$-cobordisms $\left(W^{n+1} ; M, N\right)$ which are noninertial; that is, $M$ is not diffeomorphic to $N$. (In the examples of Farrell and Hsiang, $M$ and $N$ are not even homeomorphic.) If $n \geqslant 8$, Corollary 2 implies $I_{h}(M)=I_{h}(N)$. In this example $M=L^{3} \times T^{n-3}$ where $L^{3}$ is a certain lens space and $T^{n-3}$ is the $(n-3)$-dimensional torus.

To make a nontrivial application of Corollary 2 , there must be noninertial $h$-cobordisms from $M$. It should be pointed out that there are many examples of 
inertial $h$-cobordisms. One result along these lines is given by $\mathrm{T}$. Lawson in Corollary 4 of [14]; if $n$ is even, $q$ is odd, and $\pi_{1} M^{n}=\mathbf{Z}_{q}$, then every $h$-cobordism from $M$ is inertial. In fact Proposition 1 of [14] shows that every $h$-cobordism from an even-dimensional manifold $M^{n}$ is inertial if conjugation is trivial in $\mathrm{Wh}\left(\pi_{1} M\right)$ and $L_{n+1}^{h}\left(\pi_{1} M\right)=0$. It follows from Theorem 1 of [1] or Proposition 4.2 of [3], and from Theorem 1 of [2] that these conditions hold if $\pi_{1} M$ is torsion abelian of odd order. In every dimension exceeding four, Corollaries 1.2 of [9] and 2 of [14] construct manifolds from which every $h$-cobordism is inertial. The Proposition of [8] shows that for every manifold $M^{n}, n \geqslant 5$, every $h$-cobordism is inertial after stabilizing $M$ by taking connected sum with a sufficient number of copies of $S^{p} \times S^{n-p}, 2 \leqslant p \leqslant n-2$. On the other hand, Theorem 6.1 and Lemma 6.5 of [10] construct examples of noninertial $h$-cobordisms.

\section{REFERENCES}

1. A. Bak, The involution on Whitehead torsion, Topology Appl. 7 (1977), 201-206.

2. __. Odd dimensional surgery groups of odd torsion groups vanish, Topology 14 (1975), 367-374.

3. H. Bass, $L_{3}$ of finite abelian groups, Ann. of Math. (2) 99 (1974), 118-153.

4. G. Brumfiel, Homotopy equivalences of almost smooth manifolds, Comment. Math. Helv. 46 (1971), $381-407$.

5. M. Cohen, $A$ course in simple-homotopy theory, Graduate Texts in Math., No. 10, Springer-Verlag, Berlin and New York, 1973.

6. R. DeSapio, Differentiable structures on a product of spheres, II, Ann. of Math. (2) 89 (1969), $305-313$.

7. F. Farrell and W.-C. Hsiang, h-cobordant manifolds are not necessarily homeomorphic, Bull. Amer. Math. Soc. 73 (1967), 741-744.

8. A. Hatcher and T. Lawson, Stability theorems for "concordance implies isotopy" and " $h$-cobordism implies diffeomorphism”, Duke. Math. J. 43 (1976), 555-560.

9. J.-C. Hausmann, h-cobordismes entre variétés homéomorphes, Comment. Math. Helv. 50 (1975), 9-13.

10. Open books and h-cobordisms, Comment. Math. Helv. 55 (1980), 330-346.

11. J. Hudson, Concordance, isotopy, and diffeotopy, Ann. of Math. (2) 91 (1970), 425-448.

12. Z. Janko, A new finite simple group with abelian Sylow 2-subgroups and its characterizations, J. Algebra 3 (1966), 147-186.

13. K. Kawakubo, On the inertia groups of homologv tori, J. Math. Soc. Japan 21 (1969), 37-47.

14. T. Lawson, Inertial h-cobordisms with finite cyclic fundamental group, Proc. Amer. Math. Soc. 44 (1974), 492-496.

15. J. Milnor, On manifolds homeomorphic to the 7-sphere, Ann. of Math. (2) 64 (1956), 399-405.

16. Whitehead torsion, Bull. Amer. Math. Soc. 72 (1966), 358-426.

17. R. Schultz, Smooth structures on $S^{p} \times S^{q}$, Ann. of Math. (2) 90 (1969), 187-198.

18. D. Wilkens, On the inertia groups of certain manifolds, J. London Math. Soc. (2) 9 (1975), 537-548.

Natural Sciences Division, New College of the University of South Florida, Sarasota, FLORIDA 33580 